\title{
Regularized asymptotical solutions of integro-differential systems with spectral singularities
}

\author{
Burkhan Kalimbetov ${ }^{*}$, Lesbek Tashimov, Nurlan Imanbaev and Dinmukhambet Sapakov
}

"Correspondence:

bkalimbetov@mail.ru

Department of Mathematics,

A. Yasawi International

Kazakh-Turkish University,

Turkestan, Kazakhstan

\begin{abstract}
In the work, the Cauchy problem is considered for a singularly perturbed system with zero points of the spectrum. It is proved normal solvability of general iterative problems. Unique solvability and convergence of formal solutions to the exact one are formulated.
\end{abstract}

Keywords: integro-differential equation; asymptotic convergence; regularized asymptotical solutions

\section{Introduction}

Many problems of quantum mechanics are described with singularly perturbed differential systems in the following form:

$$
\begin{aligned}
& \varepsilon \frac{d y}{d t}=A(t) y+\int_{0}^{t} \exp \left(\varepsilon^{-1} \int_{s}^{t} \mu(\theta) d \theta\right) K(t, s) y(s, \varepsilon) d s+h(t), \\
& y(0, \varepsilon)=y^{0}, \quad t \in[0, T]
\end{aligned}
$$

where $A(t), K(t, s)$ are matrices of the order $(n \times n), h(t)=\left\{h, \ldots, h_{n}\right\}$ is a given function, $y^{0} \in C^{n}$ is a constant vector, $\varepsilon>0$ is a small parameter, $\mu(t) \in C^{\infty}[0, T]$ is a scalar function. We consider the system (1.1) in the case of identical non-invertibility of the limit operator $A(t)$. This means that the degenerated system

$$
0=A(t) \bar{y}+h(t)
$$

either has not any solutions or has uncountable set of them. Therefore, we do not know in advance, to which solution of the system (1.2) tends the true solution $y(t, \varepsilon)$ of the problem (1.1) (at $\varepsilon \rightarrow+0$ ), before problems with a non-invertible limit operator $A(t)$ have been considered for differential systems [1-4], and regularized asymptotical solutions were constructed there. In the present work, integro-differential systems with quick changing kernels are considered. These systems induce additional singularities in the solution of the problem (1.1).

At first, we describe conditions at which we will consider the problem (1.1).

(1) $A(t) \in C^{\infty}\left([0, T], C^{n^{2}}\right), h(t) \in C^{\infty}\left([0, T], C^{n}\right), K(t, s) \in C^{\infty}\left(0 \leq s \leq t \leq T, C^{n^{2}}\right)$; 
(2) for any $t \in[0, T]$, the spectrum $\left\{\lambda_{j}(t)\right\}$ of the matrix $A(t)$ satisfies the following conditions:
(a) $\lambda_{i}(t) \neq 0, \lambda_{i}(t) \neq \lambda_{j}(t), i \neq j, i, j=\overline{1, p}, p<n$;
(b) $\operatorname{Re} \lambda_{j}(t) \leq 0, j=\overline{1, p}$;
(c) $\lambda_{i}(t) \equiv 0, i=\overline{p+1, n}$;

(3) $\lambda_{m}(t) \equiv \mu(t) \in C^{\infty}\left([0, T], R^{1}\right), \lambda_{m}(t) \neq \lambda_{j}(t), j=\overline{1, n} ; \forall t \in[0, T]$.

We also suppose that the matrix $A(t)$ is an operator of the simple structure (see [5]). In this case, there exists a complete system of eigenvectors $\left\{c_{j}(t)\right\}$ for the matrix $A(t)$, moreover, zero eigenvalue $\lambda_{j}(t)$ of the multiplicity $n-p$ corresponds to $n-p$ eigenvectors $c_{i}(t)$, $i=\overline{p+1, n}$. We suppose in addition that the degenerated system (1.2) has a solution. Then the right side $h(t)$ of this system has to be orthogonal to the kernel of the operator $A^{*}(t)$, i.e.,

$$
\left(h(t), d_{i}(t)\right) \equiv 0, \quad i=\overline{p+1, n}, \forall t \in[0, T]
$$

where $d_{i}(t)$ are eigenvectors of the matrix $A^{*}(t)$ corresponding to zero eigenvalue $\lambda_{i}(t) \equiv 0$ and forming the base of the kernel of the operator $A^{*}(t)$. We write the solution of the system (1.2), depending from $n-p$ arbitrary scalar functions $\alpha_{i}(t)$ defined on the segment $[0, T]$, in the following form:

$$
\bar{y}\left(t, \alpha_{p+1}, \ldots, \alpha_{n}\right)=\alpha_{p+1}(t) c_{p+1}(t)+\cdots+\alpha_{n}(t) c_{n}(t)+\tilde{y}_{0}(t)
$$

where $\tilde{y}_{0}(t)$ is a particular solution of the system (1.2) corresponding to the right side $h(t)$.

Let us compose the following system of equations $(i=\overline{p+1, n})$ :

$$
\left(-\frac{d \bar{y}\left(t, \alpha_{p+1}(t), \ldots, \alpha_{n}(t)\right)}{d t}, d_{j}(t)\right)=0 \text {, }
$$

where $\bar{y}\left(t, \alpha_{p+1}, \ldots, \alpha_{n}\right)$ is the solutions family for (1.4), $\frac{d \bar{y}}{d t}$ means the complete derivative of this family by $t$ :

$$
\frac{d \bar{y}}{d t}=\sum_{i=p+1}^{n}\left(\dot{\alpha}_{i}(t) c_{i}(t)+\alpha_{i}(t) \dot{c}_{i}(t)\right)-\dot{\tilde{y}}_{0}(t) .
$$

Let us set initial conditions for (1.5):

$$
\alpha_{i}(0)=\sum_{k=1}^{n} c_{i k}^{(-1)}(0)\left(y_{k}^{0}-\tilde{y}_{0 k}(0)\right), \quad i=\overline{p+1, n}
$$

Here, $c_{i k}^{(-1)}(0)$ are elements of the matrix $C^{-1}(t)$, which is inverse to the matrix $C(t)$ with columns $c_{i}(t) ; y_{k}^{0}$ and $\tilde{y}_{0 k}(0)$ are respectively components of the vectors $y^{0}$ and $\tilde{y}_{0}(0), i, k=$ $\overline{1, n}$. We prove that if the problem (1.5)-(1.6) has the solution $\left(\alpha_{p+1}(t), \ldots, \alpha_{n}(t)\right)$ then this solution is the limit one for the initial problem (1.1).

\section{Regularized problems}

Let us introduce regularized variables by non-zero points of the spectrum $[6,7]$

$$
\tau_{j}=\varepsilon^{-1} \int_{0}^{t} \lambda_{j}(\theta) d \theta \equiv \frac{\varphi_{j}(t)}{\varepsilon}, \quad j=\overline{1, p}
$$


and

$$
\tau_{m}=\varepsilon^{-1} \int_{0}^{t} \mu(\theta) d \theta \equiv \frac{\varphi_{m}(t)}{\varepsilon} .
$$

We consider instead of the function $y(t, \varepsilon)$ to be found for the problem (1.1) the function $\tilde{y}(t, \tau, \varepsilon)$ with more variables:

$$
\left.\tilde{y}(t, \tau, \varepsilon)\right|_{\tau=\varepsilon^{-1} \varphi(t)} \equiv y(t, \varepsilon)
$$

where $\tau=\left(\tau_{1}, \ldots, \tau_{p}, \tau_{m}\right), \varphi=\left(\varphi_{1}, \ldots, \varphi_{p}, \varphi_{m}\right)$.

Then it is natural to put the following problem for the function $\tilde{y}(t, \tau, \varepsilon)$ :

$$
\begin{aligned}
& \varepsilon \frac{\partial \tilde{y}}{\partial t}+L_{0} \tilde{y}=\int_{0}^{t} \exp \left(\varepsilon^{-1} \int_{s}^{t} \mu(\theta) d \theta\right) K(t, s) \tilde{y}\left(s, \varepsilon^{-1} \varphi(s), \varepsilon\right) d s+h(t), \\
& \tilde{y}(0,0, \varepsilon)=y^{0}
\end{aligned}
$$

where $L_{0} \equiv \lambda_{1}(t) \frac{\partial}{\partial \tau_{1}}+\cdots+\lambda_{p}(t) \frac{\partial}{\partial \tau_{p}}+\lambda_{m}(t) \frac{\partial}{\partial \tau_{m}}-A(t)$.

But the problem (2.1) is not still 'extended' for the initial problem (1.1) since we have not made regularization for the integral part. For this, we have to describe the class $M_{\varepsilon}$ asymptotically invariant (at $\varepsilon \rightarrow+0$ ) with respect to the integral operator (see [6]).

Definition We say that the function $y(t, \tau)$ belongs to the class $U$ if it can be presented as the sum:

$$
y(t, \tau)=y_{0}(t)+\sum_{j=1}^{m} y_{j}(t) e^{\tau_{j}}
$$

with coefficients $y_{j}(\tau) \in C^{\infty}\left([0, T], C^{1}\right), j=\overline{0, m}$.

If we substitute (2.2) in the integral part of the problem (2.1) and use the operation of integration by parts, then we can present integrals containing exponents in the form of the time series for $\varepsilon$ degrees:

$$
\begin{aligned}
I y(t, \tau)= & \sum_{v=0}^{\infty}(-1)^{v} \varepsilon^{v+1}\left[\left(I_{0}^{v}\left(k(t, s) y_{0}(s)\right)\right)_{s=t}\right. \\
& -\left(I_{0}^{v}\left(k(t, s) y_{0}(s)\right)\right)_{s=t} \cdot e^{\tau_{m}}+\left(I_{j}^{v}\left(k(t, s) y_{j}(s)\right)\right)_{s=t} \cdot e^{\tau_{j}} \\
& \left.-\left(I_{j}^{v}\left(k(t, s) y_{j}(s)\right)\right)_{s=0} \cdot e^{\tau_{m}}\right]+e^{\tau_{m}} \int_{0}^{t} K(t, s) y_{m}(s) d s,
\end{aligned}
$$

where $I_{0}^{0}=-\frac{1}{\mu(s)}, I_{0}^{v}=-\frac{1}{\mu(s)} \frac{\partial}{\partial s} I_{0}^{\nu-1}, I_{j}^{0}=\frac{1}{\lambda_{j}(s)-\mu(s)}, I_{j}^{v}=\frac{1}{\lambda_{j}(s)-\mu(s)} \frac{\partial}{\partial s} I_{j}^{\nu-1}, v \geq 1, j=\overline{1, p}$.

We have shown that the class $M_{\varepsilon}$ is asymptotically invariant with respect to the operator $I$. We have taken as $M_{\varepsilon}$ the restriction of the class $U$ at $\tau=\varepsilon^{-1} \varphi(t, \varepsilon)$.

Now we can write the problem extended with respect to the initial problem (1.1). It has the following form:

$$
\varepsilon \frac{\partial \tilde{y}}{\partial t}+L_{0} \tilde{y}-I \tilde{y}(t, \tau, \varepsilon)=h(t), \quad \tilde{y}(0,0, \varepsilon)=y^{0}
$$

where $I$ are integral operators given in (2.3). 
We find the solution of the problem (2.4) in the series form:

$$
y(t, \tau, \varepsilon)=\sum_{k=0}^{\infty} \varepsilon^{k} y_{k}(t, \tau)
$$

with coefficients $y_{k}(t, \tau) \in U$. If we substitute (2.5) in the system (2.4) and equate coefficients at the same degrees of $\varepsilon$, then we obtain the following problems:

$$
\begin{aligned}
& L_{0} y_{0}-I_{0} y_{0}=h(t), \quad y_{0}(0,0)=y^{0} ; \\
& L_{0} y_{1}-I_{0} y_{1}=-\frac{\partial y_{0}}{\partial t}+I_{1} y_{0}, \quad y_{1}(0,0)=0 ; \\
& \vdots \\
& L_{0} y_{k}-I_{0} y_{k}=-\frac{\partial y_{k-1}}{\partial t}+\sum_{s=0}^{k-1} I_{k-s} y_{s}, \quad y_{k}(0,0)=0, \quad k \geq 1 .
\end{aligned}
$$

\section{Solvable iterative problems}

Each of the iterative problems $\left(2.6_{i}\right), i=0, \ldots, k$ has the form:

$$
\left(L_{0}-I_{0}\right) y(t, \tau)=h(t, \tau), \quad y(0,0)=y^{0},
$$

where $h(t, \tau)$ is the corresponding right side.

We need the scalar product in the space $U$ (for any $t \in[0, T]$ ) to formulate conditions of the normal and unique solvability of the system (3.1) in $U$ :

$$
\langle y(t, \tau), z(t, \tau)\rangle \equiv\left\langle y_{0}(t)+\sum_{k=1}^{m} y_{k}(t) e^{\tau_{k}}, z_{0}(t)+\sum_{k=1}^{m} z_{k}(t) e^{\tau_{k}}\right\rangle \stackrel{\text { def }}{=} \sum_{k=0}^{m}\left(y_{k}(t), z_{k}(t)\right),
$$

where (, ) is usual scalar product in the space $U$.

The following result is valid.

Theorem 3.1 Let conditions (1), (2)(a), (2)(c), (3) hold and the right side $h(t, \tau)$ of the system (3.1) belongs to the space $U$. Then this system is solvable in the space $U$ if and only if

$$
\left\langle h(t, \tau), v_{k}(t, \tau)\right\rangle \equiv 0, \quad \forall t \in[0, T], k=\overline{1, n}
$$

where $v_{k}(t, \tau)=d_{k}(t) e^{\tau_{k}}, k=\overline{1, n}$, is the base of the kernel for the adjoint operator $L_{0}^{*}, d_{k}(t)$ are eigenvectors of the adjoint matrix $A^{*}(t)$ corresponding to eigenvalues $\overline{\lambda_{k}}(t), k=\overline{1, n}$.

Proof Let $h(t)=h_{0}(t)+\sum_{k=1}^{m} h_{k}(t) e^{\tau_{m}}$. We find the solution of the problem (3.1) in the form of the series (2.2). If we substitute (2.2) in (3.1) and equate coefficients at the same exponents and free members, then we obtain

$$
\begin{aligned}
& {\left[\lambda_{k}(t) I-A(t) y_{k}(t)\right]=h_{k}(t), \quad k=\overline{1, n},} \\
& {\left[\lambda_{m}(t) I-A(t) y_{m}(t)\right]-\int_{0}^{t} K(t, s) y_{m}(s) d s=h_{m}(t),} \\
& -A(t) y_{0}(t)=h_{0}(t),
\end{aligned}
$$


where $I$ is the unit matrix. Equation (3.4) is the integral Volterra equation of the second kind with the kernel $\left[\lambda_{m}(t) I-A(t) y_{m}(t)\right]^{-1} K(t, s)$ and the free member $\left[\lambda_{m}(t) I-\right.$ $\left.A(t) y_{m}(t)\right]^{-1} h_{m}(t)$. It is known that such equation is uniquely solvable in the class $U$. To solve the system (3.5), we transform it:

$$
y_{k}(t)=C(t) \xi(t) \equiv \sum_{k=1}^{n} \xi_{k}(t) c_{k}(t),
$$

where $\xi=\left\{\xi_{1}, \ldots, \xi_{n}\right\}$ is a new unknown vector, $C(t)=\left(c_{1}, \ldots, c_{n}\right)$ is a matrix with columns $c_{k}$, being eigenvectors of the operator $A(t)$. We have

$$
\left[\lambda_{k}(t) I-A(t)\right] C(t) \xi(t)=h_{k}(t)
$$

If we multiply from the left on $C^{-1}(t)$, then we have

$$
\left[\lambda_{k}(t)-\Lambda(t)\right]=C^{-1}(t)(t) h_{k}(t)
$$

where $\Lambda(t)=\operatorname{diag}\left(\lambda_{1}, \ldots, \lambda_{n}\right)$. Last $n-p$ components of the system (3.6) are uniquely solvable in the class $C^{\infty}[0, T]$, i.e.,

$$
y_{i}(t)=\sum_{i=p+1}^{n} \frac{\left(h_{k}(t), d_{i}(t)\right)}{\lambda_{k}(t)} c_{i}(t) .
$$

First, $p$ components of the system (3.6) are solvable in the class $C^{\infty}[0, T]$ if and only if $\left(h_{k}(t), d_{j}(t)\right) \equiv 0, j=\overline{1, p}$, what corresponds to the conditions (3.2).

Remark If the orthogonality conditions (3.2) take place, then the system (3.1) has the following solution:

$$
\begin{aligned}
y(t, \tau)= & \sum_{j=1}^{p}\left[\alpha_{j}(t) c_{j}(t)+\sum_{s=1, s \neq j}^{p} \frac{\left(h_{j}(t), d_{s}(t)\right)}{\lambda_{j}(t)-\lambda_{s}(t)} c_{s}(t)\right] e^{\tau_{j}} \\
& +\left\{\left[\lambda_{m}(t) E-A(t)\right]^{-1} h_{m}(t)\right. \\
& \left.+\int_{0}^{t} R_{m}(t, s)\left[\lambda_{m}(s) E-A(s)\right]^{-1} h_{m}(s) d s\right\} e^{\tau_{m}}+\sum_{i=p+1}^{n} \alpha_{i}(t) c_{i}(t),
\end{aligned}
$$

where $\alpha_{j}(t)$ are arbitrary functions from the class $C^{\infty}[0, T], j=\overline{1, p}, R_{m}(t, s)$ is the resolvent of the kernel $\left[\lambda_{m}(s) E-A(s)\right]^{-1} h_{m}(s), E$ unit matrix.

Unique solvability for the system (3.1) follows from the following proposition.

Theorem 3.2 Let conditions of Theorem 3.1 take place and the right side $h(t, \tau) \in U$ of the system (3.1) satisfies the orthogonality conditions (3.2). Then the system (3.1) has the unique solution in the space $U$ at additional conditions:

$$
\left\langle-\frac{\partial y}{\partial t}+I_{1} y+Q(t, \tau), y_{k}(t, \tau)\right\rangle \equiv 0, \quad \forall t \in[0, T], k=\overline{1, n}
$$

where $Q(t, \tau)$ is the known function. 
Constructing solutions $y_{0}(t, \tau), \ldots, y_{N}(t, \tau)$ of the problems $\left(2.6_{1}\right), \ldots,\left(2.6_{N}\right)$ in the space $U$ we compose the partial sum

$$
S_{N}(t, \tau, \varepsilon)=\sum_{k=0}^{N} \varepsilon^{k} y_{k}(t, \tau) \text {. }
$$

We denote the restriction of this sum at $\tau=\varepsilon^{-1} \varphi(t)$ as $y_{\varepsilon N}(t)$. The following result takes place.

Theorem 3.3 Let conditions (1)-(3) hold. Then the initial problem (1.1) has at sufficiently small $\varepsilon \in\left(0, \varepsilon_{0}\right]$ the unique solution $y(t, \varepsilon)$ in the class $C^{1}\left([0, T], C^{n}\right)$; moreover, the following estimate is valid:

$$
\left\|y(t, \varepsilon)-y_{\varepsilon N}(t)\right\|_{C[0, T]} \leq C_{N} \varepsilon^{N+1} \quad(N=0,1, \ldots)
$$

where the constant $C_{N}>0$ does not depend on $\varepsilon$ at $\left(0, \varepsilon_{0}\right]\left(\varepsilon_{0}>0\right.$ is sufficiently small).

Competing interests

The authors declare that they have no competing interests.

\section{Authors' contributions}

All authors read and approved the final manuscript.

Received: 28 January 2013 Accepted: 3 April 2013 Published: 18 April 2013

\section{References}

1. Myagkova, MP: Asymptotical solution of the weakly non-linear boundary value problem with a degenerated limit operator. In: The All-Union Conference on Asymptotical Methods. Part II, pp. 88-90. Nauka, Alma-Ata (1979) (in Russian)

2. Lomov, IS: Regularization of singular disturbances on the spectrum of the limit operator. Vestn. Mosk. Univ., Mat. Mekh. 31, 6-13 (1976) (in Russian)

3. Safonov, VF: Asymptotical solution of a singular disturbed problem with nonzero point of the limit operator spectrum. Proc. MEl 357, 95-97 (1978) (in Russian)

4. Erdogan, F: An exponentially fitted method for singularly perturbed delay differential equations. Adv. Differ. Equ. 2009, Article ID 781579 (2009)

5. Gantmakher, FG: Matrices Theory, p. 530. Nauka, Moscow (1987) (in Russian)

6. Lomov, SA: Introduction in the General Theory of Singular Perturbation. Translations of Mathematical Monographs, vol. 112. Am. Math. Soc, Providence (1992)

7. Kalimbetov, BT, Temirbekov, MA, Khabibullayev, ZO: Asymptotic solutions of singular perturbed problems with an instable spectrum of the limiting operator. Abstr. Appl. Anal. 2012, Article ID 120192 (2012)

doi:10.1186/1687-1847-2013-109

Cite this article as: Kalimbetov et al.: Regularized asymptotical solutions of integro-differential systems with spectral singularities. Advances in Difference Equations 2013 2013:109. 